\title{
Influence of maintained ocular deviation on the spatial displacement component of the oculogyral illusion
}

\author{
JOHN N. EVANOFF and JAMES R. LACKNER \\ Brandeis University, Waltham, Massachusetts
}

\begin{abstract}
We evaluated the effect of maintained gaze deviation on the displacement component of the oculogyral illusion (OGI). Whiteside, Graybiel, and Niven (1965) proposed that the perception of an OGI was dependent upon the central monitoring of a fixation command needed to suppress vestibular nystagmus and maintain a retinally stable target. By employing a procedure developed by Alexander (1922), we varied the fixation signal strength needed to maintain stable target fixation during rotation, and found systematic and predictable differences in the magnitude of the displacement component of the OGI. These results suggest that the visual fixation signal needed to override a covert vestibular nystagmus is used in computing the visual direction of a target, and provide support for the theoretical analysis of the OGI proposed by Whiteside, Graybiel, and Niven.
\end{abstract}

In the dark, exposure to continuous rotary acceleration about the vertical $(Z)$ body axis results in a characteristic pattern of eye movements known as vestibular nystagmus. Nystagmus can be effectively suppressed during low levels of acceleration by fixation of a target light on the rotation device, stationary with respect to the body. Suppression of nystagmus with such fixation of a target light is evident over acceleration rates from approximately $1 \% \mathrm{sec}^{2}$ to $30 \% \mathrm{sec}^{2}$. Under these circumstances, an efferent command must be used to innervate extraocular muscles antagonistic to those involved in production of the slowphase component of nystagmus. To achieve effective suppression, the magnitude of the fixation command must equal the involuntary, reflexive slow-phase velocity. During suppresion of nystagmus, despite the absence of demonstrable ocular deviations exceeding a few minutes of arc (Byford, 1963), the visual target that is stationary in relation to the observer will be seen by him/her to undergo a smooth unidirectional motion and displacement in relation to the observer's body in the direction of acceleration. This phenomenon is known as the "oculogyral illusion" (OGI) (Graybiel \& Hupp, 1946). The magnitude of the apparent displacement may attain $20^{\circ}-30^{\circ}$.

Whiteside, Graybiel, and Niven (1965) have suggested that the visual-displacement component of the OGI in the direction of body acceleration may result from a monitoring by the central nervous system of the fixation command necessary to suppress vestibular nystagmus and thereby maintain a retinally stable target. In this context, it should be emphasized that the OGI has two dissociable

The authors wish to thank Paul DiZio for developing the computer programs used in the analysis of eye movement data. Support for this research was provided by NASA Contract NAS9-15147. Reprint requests should be addressed to J. N. Evanoff, Graybiel Spatial Orientation Laboratory, Brandeis University, 415 South Street, Waltham, MA 02254. components: apparent target displacement in relation to the observer's midline and apparent motion of the target in relation to external space. The angular accelerations at which target motion is experienced are lower than those at which apparent displacement is perceived (see Lackner, 1976). Many studies of the OGI either fail to recognize that it has two components or focus only on the apparent motion component (e.g., Clark \& Stewart, 1969). The present study was concerned exclusively with the observer-relative displacement of the OGI.

If Whiteside et al. (1965) are correct in their explanation of the OGI, and their view has recently been extended by Evanoff (1984) and Post and Leibowitz (1985), varying the fixation-signal strength necessary to maintain stable target fixation during acceleration should result in systematic and predictable differences in the magnitude of the illusion. It is known that vestibular nystagmus in the dark can be influenced by voluntarily maintaining leftward or rightward gaze during rotation (Evanoff \& Lackner, 1986). Deviation of the eyes in the direction of the fast-phase component of nystagmus enhances slow-phase amplitude, slow-phase velocity, and fast-phase amplitude of per-rotary nystagmus. The farther the commanded position of the eyes is from the objective median plane in the direction of the fast phase, the greater the amplitude and velocity of the resulting nystagmus. Deviation of gaze in the direction of the slow phase tends to suppress the intensity of vestibular nystagmus. This pattern is consistent with the early investigations of Alexander (1922), who used the character of postrotary nystagmus as a means of assessing the integrity of vestibular and cerebellar function. Alexander had subjects fixate a stationary point placed in different positions relative to the direction of their prior rotation. Voluntary deviation of gaze in the direction of prior rotation diminished the amplitude and frequency of postrotary nystagmus; deviations of the eyes 
in the opposite direction augmented postrotary nystamus. This pattern of influence has become known as "Alexander's law." The findings of Evanoff and Lackner described above indicate that Alexander's law also holds for prerotary vestibular nystagmus and provides a way to manipulate the strength of the fixation signal needed to override a vestibular nystagmus. Such variations can be achieved simply by placement of the visual target to the left or the right of the observer's midline.

\section{METHOD}

\section{Observers}

Eight observers were paid for their voluntary participation. Two of them had experienced the OGI or similar visual illusions prior to this experiment. All had normal vestibular function.

\section{Apparatus}

Labyrinthine stimulation was provided in all conditions by means of a rotating chair that permitted precise alignment of the $Z$-axis of the subject's head and torso with the axis of chair rotation (see Figure 1).

\section{Procedure}

Six conditions were used to assess the influence of maintained ocular deviation on magnitude indications of the displacement component of the OGI. In these conditions, the subject was exposed to clockwise $(\mathrm{CW})$ and counterclockwise $(\mathrm{CCW})$ trapezoidal ve-

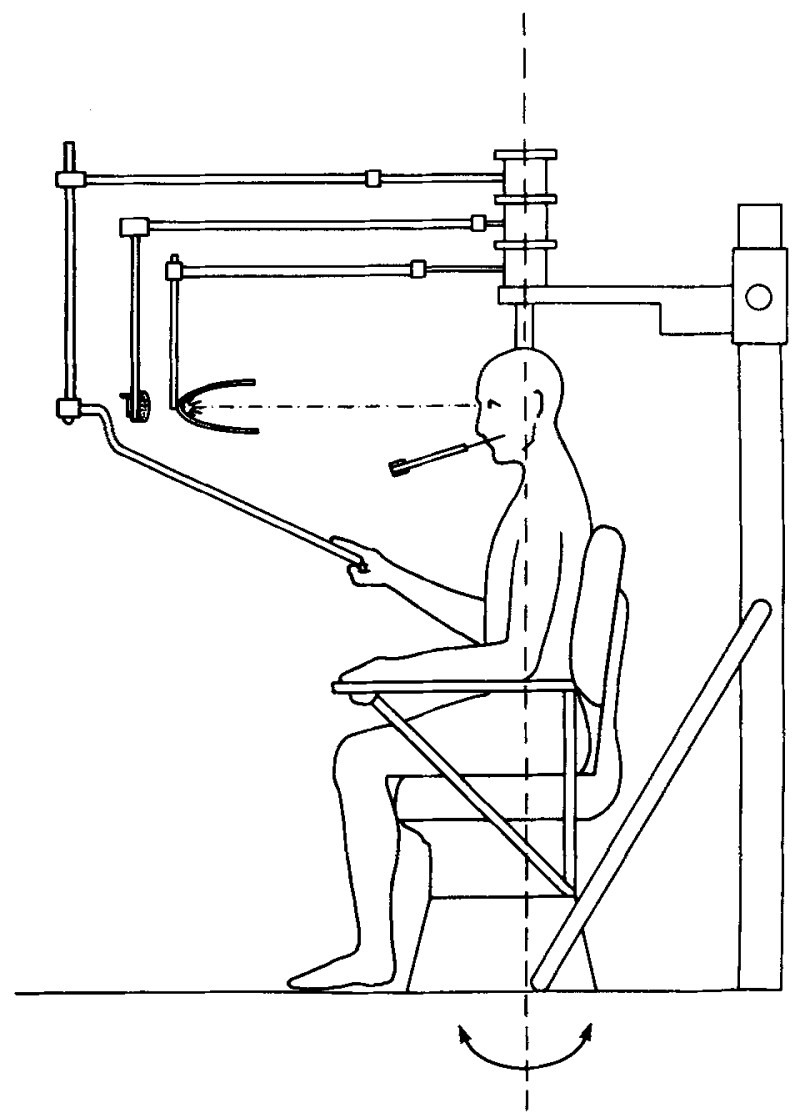

Figure 1. Schematic representation of the rotating chair used to provide labyrinthine stimulation. locity profiles, with his/her longitudinal $(Z)$ body axis aligned with the axis of chair rotation. The subject was instructed to fixate a single target light (ultraminiature LED, . $1^{\circ}$ visual angle) that remained visible throughout the entire velocity profile and a 30 -sec period immediately following deceleration. The target light was positioned either in alignment with an extension of the subject's objective median plane or $21^{\circ}$ left or right of that plane. The subject's head was held, ventriflexed $25^{\circ}$ in alignment with the body's objective median plane, by means of a dental acrylic bite plate assembly. This head position also placed the horizontal semicircular canals approximately in the plane of rotation. The conditions were run in complete darkness, with the exception of the target light, and assumed the following form: Condition 1-Eyes aligned with objective median plane, $\mathrm{CW}$ rotation, $15 \% / \mathrm{sec}^{2}$. Condition $2-$ Eyes deviated $21^{\circ}$ right, $\mathrm{CCW}$ rotation, $15^{\circ} / \mathrm{sec}^{2}$. Condition 3-Eyes deviated $21^{\circ}$ left, $\mathrm{CW}$ rotation $15^{\circ} / \mathrm{sec}^{2}$. Condition 4-Eyes deviated $21^{\circ}$ left, CCW rotation, $15^{\circ} / \mathrm{sec}^{2}$. Condition 5-Eyes deviated $21^{\circ}$ right, $\mathrm{CW}$ rotation, $15 \% / \mathrm{sec}^{2}$. Condition 6-Eyes aligned with objective median plane $\mathrm{CCW}$ rotation, $15^{\circ} / \mathrm{sec}^{2}$.

All subjects participated in all conditions in a repeated measures design. The conditions were presented in random order by means of a modified Greco-Latin square, such that no condition followed any other condition more than once within and across all subjects. An acceleration and deceleration rate of $15 \% / \mathrm{sec}^{2}$ was used in each condition and was separated by a constant-velocity interval of $45 \mathrm{sec}$. The duration of acceleration was set at $6.67 \mathrm{sec}$ to achieve a constant velocity of $100^{\circ} / \mathrm{sec}$. Between trials, a 5-min rest interval provided time for recalibration of eye position and subjective reports to be obtained. During each condition, the subject's task was to fixate the target light and point to its perceived spatial location with an indicator rod grasped lightly in his/her right hand (see Figure 1). Pointing began approximately $10 \mathrm{sec}$ prior to the onset of acceleration and continued until approximately $30 \mathrm{sec}$ after chair movement ceased. This provided an indication of perceived subjectrelative, target displacement, in degrees of visual arc, for each phase of the trapezoidal velocity profile and a poststimulation period. We know from other experiments in which we have measured the influence of angular acceleration on pointing movements that subjects can point accurately to body-relative targets using the present pointing procedure (Evanoff \& Lackner, in press). The pointing task was used in the present experiment to allow the time course of the OGI to be monitored.

Conjugate horizontal eye position was monitored continuously throughout each trial by means of conventional, dc-coupled, electrooculography. The electrodes (Harco HAR-157, silver/silverchloride, pregelled) were positioned on the lateral canthus of each eye with a neutral/ground electrode positioned on the forehead. The EOG signals were amplified before being fed through the chair's commutator assembly to a polygraph and to a Hewlett-Packard FM instrumentation recorder (Model 3968A) for subsequent analog-todigital (A/D) conversion and computer analysis. At the beginning of each trial, a code signal was automatically superimposed on the eye position channel by the chair microprocessor to synchronize the onset of computer analysis with the onset of chair acceleration. Chair velocity, the position signal from the indicator rod, and experimenter comments were also recorded on separate tape channels. The subject was dark-adapted for $10 \mathrm{~min}$ prior to the start of a condition.

\section{Data Transformations}

The experimental records of indicated target-light position were computer sampled at a rate of $20 \mathrm{~Hz}$ during A/D conversion. The average indicated position of the target, in degrees, was then established for each level of the chair velocity profile and a 30-sec postrotary interval. Prior to statistical analysis, a $21^{\circ}$ constant was subtracted from the positional data obtained from those conditions that involved a maintained $21^{\circ}$ ocular deviation. This was done to nor- 
malize these data for comparison with the standard conditions in which the target light was physically aligned with the subject's midline. The values from the conditions in which the eyes were deviated in the direction of the fast-phase component of nystagmus were combined (CCW acceleration, eyes left with $\mathrm{CW}$ acceleration, eyes right). Similarly, values from the conditions in which the eyes were deviated in the slow-phase direction were combined (CCW acceleration, eyes right with $\mathrm{CW}$ acceleration, eyes left). The standard conditions (CW and $\mathrm{CCW}$ ) were also combined.

\section{RESULTS}

During the acceleration phase in each condition, the subjects indicated a target displacement in the direction of rotation. Although this displacement diminished during the period of constant velocity, none of the subjects indicated that the target light had returned to its initial position prior to the onset of deceleration. A two-way analysis of variance was performed on these data.

The eye-deviation conditions and the control conditions were found to be significantly different from one another $[F(2,10)=19.170, p=.0004]$. No differences were present between the acceleration and constant-velocity levels of any trapezoidal velocity profile $[F(1,5)=6.203$, $p=.055]$. A Scheffé test of planned comparisons revealed that the conditions in which the eyes were deviated in the direction of the fast phase of the nystagmus (that would have occurred if the fixation target had been absent) and the conditions in which the eyes were deviated in the direction of the slow phase were significantly different from one another $(p<.01)$. The OGI displacement during maintained gaze deviation in the direction of the slow-phase component of latent nystagmus was significantly smaller than that of the standard $(p<.01)$ and significantly larger than that of the standard when the eyes were deviated in the direction of the fast-phase component $(p<.01)$ (see Figure 2).

In their subjective reports, each subject described having seen smooth motion and displacement of the target. Analysis of the EOG records indicated that none of the subjects produced ocular deviations from the fixation target that exceeded $2^{\circ}$. Nevertheless, in other subjective reports, the subjects said they had perceived that their eyes made large nystagmus-like movements although there was no blurring of the target light and they experienced no difficulty in maintaining fixation during any portion of the velocity profile. Subjects also reported higher perceived target velocities under conditions in which the eyes were deviated in the direction of the fast-phase component of nystagmus.

\section{DISCUSSION}

According to Whiteside et al. (1965), the OGI may depend upon the central monitoring of a fixation command needed to "override"' reflexive, compensatory eye deviations so as to maintain stable retinal images. This efferent fixation command, elicited by retinal error information, is provided to the extraocular muscles to prevent the production of the slow-phase component of vestibular nystagmus. According to this viewpoint, in computations of the visual direction of the target light, the vestibularly driven oculomotor signals that result in nystagmus are ignored and, instead, the fixation command necessary to stabilize the eyes is monitored. Consequently, the subject perceives motion of the target in the direction of acceleration. There are two components to this motion: an

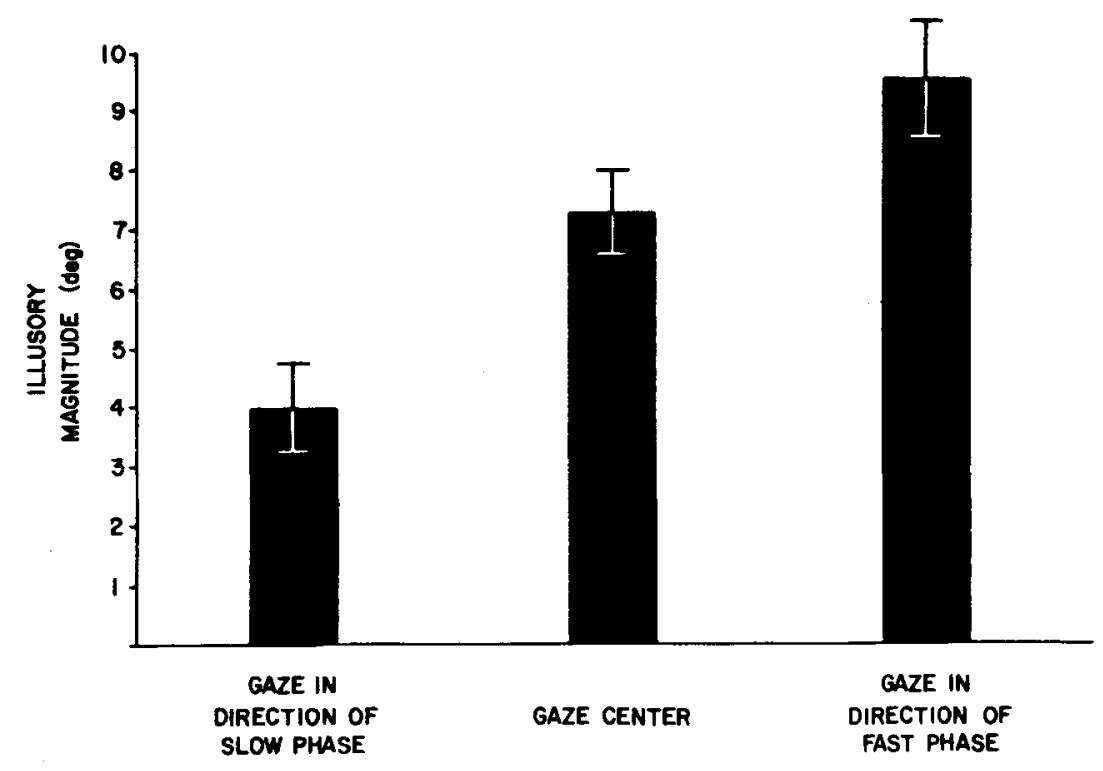

Figure 2. Magnitude indications of OGI displacement during maintained gaze deviation in the direction of the slow-phase component of latent nystagmus, in the direction of the fast-phase component of latent nystagmus, or in alignment with the objective median plane of the body. (Error bars represent 1 standard error of the mean.) 
apparent displacement of the target in relation to the subject's midline and an apparent velocity of the target with regard to unseen inertial space. The apparent displacement is thought to be related to the magnitude of the fixation signal available to suppress the slow-phase deviation of the eyes and the apparent velocity signal to be related to the subject's perception of body rotation.

If this theory is correct, it should be possible to alter the magnitude of the apparent displacement component of the OGI by manipulating the strength of the fixation command needed to suppress vestibular nystagmus. We accomplished this in the present experiment by making use of the observation that normal vestibular nystagmus in the dark can be systematically altered by voluntary deviation of the eyes in the direction of the fast- or slow-phase components. Accordingly, under conditions requiring fixation of a deviated target light during acceleration, the fixation reflex activity needed to maintain a foveated image should vary by an amount proportional to the slowphase amplitude of the covert vestibular nystagmus. We expected such changes in activity to modify accordingly the displacement component of the OGI, and our findings supported these expectations.

With the target light deviated $21^{\circ}$ from the subject's midline, in the direction of acceleration, the displacement component of the OGI was increased $33 \%$ beyond the straight-ahead-fixation standard condition. Deviation of the eyes in the direction opposite acceleration resulted in a $45 \%$ decrease from that of the standard condition. At the onset of deceleration, there was a comparable and proportionate diminution of the indicated magnitude of the OGI. This pattern of changes reflects similar changes we observed (Evanoff \& Lackner, 1986) in slow-phase amplitude of vestibular nystagmus in the dark using an identical acceleration rate. In that study, average slowphase amplitude was increased by $32 \%$ when the eyes were deviated $21^{\circ}$ in the direction of acceleration and decreased $13 \%$ when the eyes were deviated opposite the direction of acceleration. This pattern suggests that a visual fixation signal to override a covert nystagmus is directly involved in computing the visual direction of the OGI target. Accordingly, this pattern provides support for the theoretical analysis of the OGI postulated by Whiteside et al. (1965), Evanoff (1984), and Post and Leibowitz (1985).

All of the subjects in the present experiment spontaneously reported, after each condition, that it felt as if their eyes had undergone large nystagmus-like deviations even though the target did not blur. During the trials, their eyes were actually maintaining quite accurate fixation of the target. This finding suggests that perceived ocular position can be influenced by reflexly commanded but not expressed ocular deviations. It is in accord with earlier observations indicating that perceived visual direction and perceived eye positions can be disoriented when proprioceptive misinformation is provided about the location of a visual target that is being steadily fixated (Lackner \& Levine, 1978; Lackner \& Shenker, 1985).

\section{REFERENCES}

Alexander, G. (1922). Bau und Funktion des perilymphatischen Gewebes im inneren Ohr des Menschen und der höheren Säugetiere. Archives Neerlandaises de Physiologie, 7, 552-557.

ByForD, G. H. (1963). Eye movements and the optogyral illusion. Aerospace Medicine, 34, 119-123.

Clark, B., \& StEWART, J. D. (1969). Effects of angular acceleration on man: Thresholds for the perception of rotation and the oculogyral illusion. Aerospace Medicine, 40, 952-956.

EVANOFF, J. N. (1984). Some influences of brachial proprioception and compensatory reflexes on oculomotor control and visual direction during labyrinthine stimulation. (Doctoral disseration, Brandeis University, 1984). University Microfilms Intemational No. 84-20,764.

EvanofF, J. N., \&ACKNER, J. R. (1986). Influence of voluntary ocular deviation on vestibular nystagmus. Acta Oto-Laryngologica (Stockholm), 102, 450-456.

EvANOFF, J. N., \& LACKNER, J. R. (in press). Some proprioceptive influences on the spatial displacement component of the oculogyral illusion (OGI). Perception \& Psychophysics.

Graybiel, A., \& HuPP, D. (1946). The oculo-gyral illusion: A form of apparent motion which may be observed following stimulation of the semi-circular canals. Joumal of Aviation Medicine, 17, 1-12.

LACKNER, J. R. (1976). Influence of abnormal postural and sensory conditions on human sensorimotor localization. Environmental Biology \& Medicine, 2, 139-177.

LACKNER, J. R., \& Levine, M. S. (1978). Visual direction depends on the operation of spatial constancy mechanisms. Neuroscience Letters, 7, 207-212.

LaCkner, J. R., Shenker, B. (1985). Proprioceptive influences on auditory and visual spatial localization. Journal of Neuroscience, 3 , 579-583.

Post, R. B., Leibowitz, H. W. (1985). A revised analysis of the role of efference in motion perception. Perception, 14, 631-643.

Whiteside, T. C. D., Graybiel, A., \& Niven, J. I. (1965). Visual illusions of movements. Brain, 88, 193-219.

(Manuscript received April 10, 1986; revision accepted for publication January 12,1987 .) 